\title{
Short-term outcomes of lateral lumbar interbody fusion without decompression for the treatment of symptomatic degenerative spondylolisthesis at L4-5
}

\author{
Peter G. Campbell, MD, ${ }^{1}$ Pierce D. Nunley, MD, ${ }^{2}$ David Cavanaugh, MD, ${ }^{1}$ Eubulus Kerr, MD, ${ }^{2}$ \\ Philip Andrew Utter, MD, ${ }^{1}$ Kelly Frank, MS, ${ }^{3}$ and Marcus Stone, $\mathrm{PhD}^{3}$ \\ Departments of ${ }^{1}$ Neurosurgery, ${ }^{2}$ Orthopedics, and ${ }^{3} \mathrm{Clinical}$ Research, Spine Institute of Louisiana, Shreveport, Louisiana
}

\begin{abstract}
OBJECTIVE Recently, authors have called into question the utility and complication index of the lateral lumbar interbody fusion procedure at the L4-5 level. Furthermore, the need for direct decompression has also been debated. Here, the authors report the clinical and radiographic outcomes of transpsoas lumbar interbody fusion, relying only on indirect decompression to treat patients with neurogenic claudication secondary to Grade 1 and 2 spondylolisthesis at the L4-5 level.
\end{abstract}

METHODS The authors conducted a retrospective evaluation of 18 consecutive patients with Grade 1 or 2 spondylolisthesis from a prospectively maintained database. All patients underwent a transpsoas approach, followed by posterior percutaneous instrumentation without decompression. The Oswestry Disability Index (ODI) and SF-12 were administered during the clinical evaluations. Radiographic evaluation was also performed. The mean follow-up was 6.2 months.

RESULTS Fifteen patients with Grade 1 and 3 patients with Grade 2 spondylolisthesis were identified and underwent fusion at a total of 20 levels. The mean operative time was 165 minutes for the combined anterior and posterior phases of the operation. The estimated blood loss was $113 \mathrm{ml}$. The most common cage width in the anteroposterior dimension was $22 \mathrm{~mm}(78 \%)$. Anterior thigh dysesthesia was identified on detailed sensory evaluation in 6 of 18 patients (33\%); all patients experienced resolution within 6 months postoperatively. No patient had lasting sensory loss or motor deficit. The average ODI score improved 26 points by the 6-month follow-up. At the 6 -month follow-up, the SF-12 mean Physical and Mental Component Summary scores improved by $11.9 \%$ and $9.6 \%$, respectively. No patient required additional decompression postoperatively.

CONCLUSIONS This study offers clinical results to establish lateral lumbar interbody fusion as an effective technique for the treatment of Grade 1 or 2 degenerative spondylolisthesis at L4-5. The use of this surgical approach provides a minimally invasive solution that offers excellent arthrodesis rates as well as favorable clinical and radiological outcomes, with low rates of postoperative complications. However, adhering to the techniques of transpsoas lateral surgery, such as minimal table break, an initial look-and-see approach to the psoas, clear identification of the plexus, minimal cranial caudal expansion of the retractor, mobilization of any traversing sensory nerves, and total psoas dilation times less than 20 minutes, ensures the lowest possible complication profile for both visceral and neural injuries even in the narrow safe zones when accessing the L4-5 disc space in patients with degenerative spondylolisthesis.

https://thejns.org/doi/abs/10.3171/2017.10.FOCUS17566

KEY WORDS XLIF; DLIF; LLIF; extreme lateral interbody fusion; lateral lumbar interbody fusion; direct lateral interbody fusion; lateral fusion; spondylolisthesis; transpsoas

$\mathrm{D}$ EGENERATIVE spondylolisthesis (DS) is often characterized by disc and facet degeneration that allows sagittal translation of the involved segment, commonly resulting in lateral recess and/or foraminal stenosis. ${ }^{17}$ It disproportionally affects older females at the L4-5 level and is often associated with more sagittally oriented facet joints. ${ }^{7}$ Treatment options have ranged from limited decompression to decompression with anterior and/or posterior fusion with or without listhesis correction to multilevel fusion for deformity correction. Recent randomized controlled trials have shown clinically significant improvement in patients with spondylolisthesis who

ABBREVIATIONS DS = degenerative spondylolisthesis; LLIF = lateral lumbar interbody fusion; MCS = Mental Component Summary; ODI = Oswestry Disability Index; PCS = Physical Component Summary. 
undergo treatment not only with surgical procedures in general but, more specifically, with the addition of fusion to the procedure..$^{8,33}$

Recent comparative analyses have evaluated a variety of fusion procedures in treating DS at the L4-5 level, including anterior lumbar interbody fusion, posterior lumbar interbody fusion, transforaminal lumbar interbody fusion, and lateral lumbar interbody fusion (LLIF) ${ }^{16}$ Despite a great deal of investigation, no approach has clinically been proven more effective than the others, and no universal treatment guidelines have been proposed. ${ }^{26}$

Often, anterior lumbar interbody fusion approaches are associated with a significant degree of difficulty with exposure. This is generally due to difficulty with venous mobilization or retroperitoneal scarring, whereas disruption of the lumbar paraspinal musculature and the nerve root injury secondary to cage insertion are problematic complications of the posterior lumbar interbody fusion and transforaminal lumbar interbody fusion procedures. ${ }^{19}$ Because of its ability to provide indirect decompression without disrupting the posterior elements, segmental stability, and lordosis restoration, the transpsoas LLIF technique has been proposed as a treatment option for DS at L4-5.,11,15,25,27 However, concerns exist about the rate of neurological complications associated with the lateral approach. .,10,14,24 $^{2}$ Furthermore, these complications are most conspicuous at the L4-5 level due to the anatomical arrangement of the lumbar plexus with narrower safe working zones more inferiorly in the lower lumbar spine. ${ }^{30}$ Anterolisthesis at the L4-5 level serves to exacerbate this risk. ${ }^{27}$ The purpose of this study is to evaluate the clinical and radiographic outcomes of utilizing LLIF for the indication of DS at the L4-5 level.

\section{Methods}

A retrospective review of a prospectively maintained database was performed after institutional review board approval was received. All patients had undergone unsuccessful conservative treatment for at least 6 months or exhibited worsening neurological symptoms during the conservative management period. Outcomes data were collected at a single institution with multiple spine surgeons. Inclusion criteria for the study were consecutive patients with Grade 1 or Grade 2 degenerative spondylolisthesis at L4-5 treated with a 1- or 2-level LLIF procedure between July 1, 2016, and July 1, 2017. Patients meeting these criteria were included; there were no exclusions. Outcomes were collected preoperatively and at standard follow-up intervals: 2 and 6 weeks, and 3, 6, and 12 months postoperatively, as applicable. All other fusion indications were excluded (degenerative disc disease, mechanical collapse, adjacent-segment disease, and degenerative scoliosis). Eighteen consecutive patients were identified for inclusion in the study. All patients presented with back and leg symptomatology.

\section{Surgical Technique}

All patients underwent LLIF to the L4-5 disc space via a transpsoas approach for the treatment of spondylolisthesis. Based on surgeon preference, either titanium or polyetheretherketone intervertebral cages were used. Implant- ed cages varied between $7^{\circ}$ and $10^{\circ}$ of lordosis, and graft material also varied by surgeon preference. Neurophysiological monitoring was performed in all cases. All cases relied on indirect decompression, and the use of supplemental posterior fixation with pedicle screws was standard practice. Unilateral fixation was only considered as an option in conjunction with the placement of a $26-\mathrm{mm}$ cage.

Every patient underwent specific evaluation for anterior thigh dysesthesia at each clinical encounter, with a complete sensory evaluation of light touch and pinprick in both lower extremities. Motor function testing was also performed at each visit, which encompassed testing of 5 myotomes from L-2 to S-1. Reflex function in the knee and ankle was also evaluated both pre- and postoperatively.

Patient outcomes; complications, including plexopathy (even if transient); Oswestry Disability Index (ODI) scores; and scores for both components (mental and physical) of the SF-12 were evaluated. Substantial clinical benefit thresholds for the ODI were defined as a 15-point improvement in the ODI score. The SF-12 is a multipurpose shortform survey with 12 questions, all selected from the SF-36 Health Survey. Any improvement in the components of the SF-12, which would be represented by a higher score, was considered significant.

Radiographic assessments were made using standing lumbar lateral radiographs at preoperative evaluation and on the date of last clinical follow-up. Evaluation was performed to assess overall changes in lumbar lordosis at pre- and postoperative intervals. Lumbar lordosis was measured as the angulation from the superior endplate of L-1 to the superior endplate of S-1. Slip measurements associated with postoperative reductions were performed on calibrated lateral standing radiographs at the L4-5 level (Sectra IDS7, Sectra AB). Any endplate subsidence was also noted.

\section{Results}

Eighteen consecutive patients were included in this study cohort. The mean age at the time of surgery was 64 years, and $61 \%$ of patients were female. The L4-5 level was treated in isolation in $89 \%$, and $11 \%$ of patients underwent an L3-4 and L4-5 LLIF. Twenty levels were treated in total. Slip was characterized as Grade 1 in $83 \%$ of patients and Grade 2 in $17 \%$. The mean follow-up time was 6.2 months, with a range of 3-13 months.

Posterior instrumentation was placed with bilateral pedicle screws in $89 \%$ of patients and a unilateral pedicle screw construct in $11 \%$. All procedures were performed in the traditional direct lateral position and then repositioned for the posterior approach. Biplane fluoroscopy was employed for placement of all pedicle screws. No patient underwent additional open or endoscopic central or foraminal decompression posteriorly. The mean operative time was 165 minutes for the combined anterior and posterior phases of the operation. The mean estimated blood loss was 113 $\mathrm{ml}$. The most common cage width in the anteroposterior dimensions at the L4-5 level was $22 \mathrm{~mm}$ (78\%), followed by $18 \mathrm{~mm}$ and $26 \mathrm{~mm}$, each with an $11 \%$ utilization rate. Complete demographic and treatment data are detailed in Table 1. 
TABLE 1. Baseline demographic and treatment data from patients undergoing LLIF for the treatment of spondylolisthesis

\begin{tabular}{lc}
\hline \multicolumn{1}{c}{ Variable } & Value \\
\hline No. of patients & 18 \\
\hline Age in yrs, mean \pm SD & $64 \pm 10.5$ \\
\hline BMl in $\mathrm{kg} / \mathrm{m}^{2}$, mean \pm SD & $34 \pm 7$ \\
\hline Female, $\mathrm{n}(\%)$ & $11(61)$ \\
\hline Grade of spondylolisthesis, $\mathrm{n}(\%)$ & $15(83)$ \\
\hline 1 & $3(17)$ \\
\hline 2 & $2(11)$ \\
\hline Levels treated, $\mathrm{n}(\%)$ & $16(89)$ \\
\hline L3-4 \& L4-5 & $100 \%$ \\
\hline L4-5 & $0 \%$ \\
\hline Posterior instrumentation & $113 \pm 79$ \\
\hline Additional decompression & $165 \pm 58$ \\
\hline EBL in ml, mean \pm SD & \\
\hline Op in mins, mean \pm SD & $2(11)$ \\
\hline Cage width at L4-5 in mm, $\mathrm{n}(\%)$ & $14(78)$ \\
\hline 18 & $2(11)$ \\
\hline 22 & $6.2 \pm 2.7$ \\
\hline 26 & \\
\hline Follow-up in mos, mean \pm SD & \\
\hline
\end{tabular}

$\mathrm{BMI}=$ body mass index; $\mathrm{EBL}=$ estimated blood loss.

Radiographic evaluation identified a mean preoperative global lordosis as $60.3^{\circ}$ and a mean postoperative global lordosis of $62.4^{\circ}$. Complete reduction of spondylolisthesis
$(<1 \mathrm{~mm})$ occurred in 13 of 18 patients (72\%) (Fig. 1). Of those patients who had a complete reduction of listhesis postoperatively, 11 patients initially had Grade 1 and 2 patients had Grade 2 spondylolisthesis. Of the remaining 5 patients without postoperative resolution of the slip, a $43 \%$ reduction in slip size was noted. Four of 5 of these patients had Grade 1 spondylolisthesis preoperatively. One patient was noted radiographically to have $20 \%$ subsidence of the lateral cage into the superior endplate of L-5. This finding was not clinically significant.

There were no intraoperative complications. No patient required hospital readmission during the 90 -day perioperative period. One patient (6\%) experienced atrial fibrillation postoperatively and underwent treatment in an outpatient setting. Anterior thigh dysesthesia was identified on detailed sensory evaluation in 6 of 18 patients (33\%). All cases resolved between 2 weeks and 6 months postoperatively. Thigh dysesthesia contralateral to the exposure was present in 1 case, with the remaining 5 patients reporting sensory loss ipsilateral to the side of exposure and psoas manipulation. One patient also had mild transient hip flexor weakness that resolved by 4 weeks postoperatively. There were no cases of hardware failure or pseudarthrosis that required additional surgical intervention.

From preoperatively to the 3-month follow-up, the mean ODI score improved from 49.1 to 34.5 (29.7\%). At the 6-month follow-up time point, the ODI score continued to improve to 23.1 (53.0\%; Fig. 2). A marked reduction in ODI score was seen at the 12-month follow-up, but this was graphically excluded, as this metric was only available for 2 patients in the study population. The mean follow-up time was 6.2 months, with a range of 3-13 months.

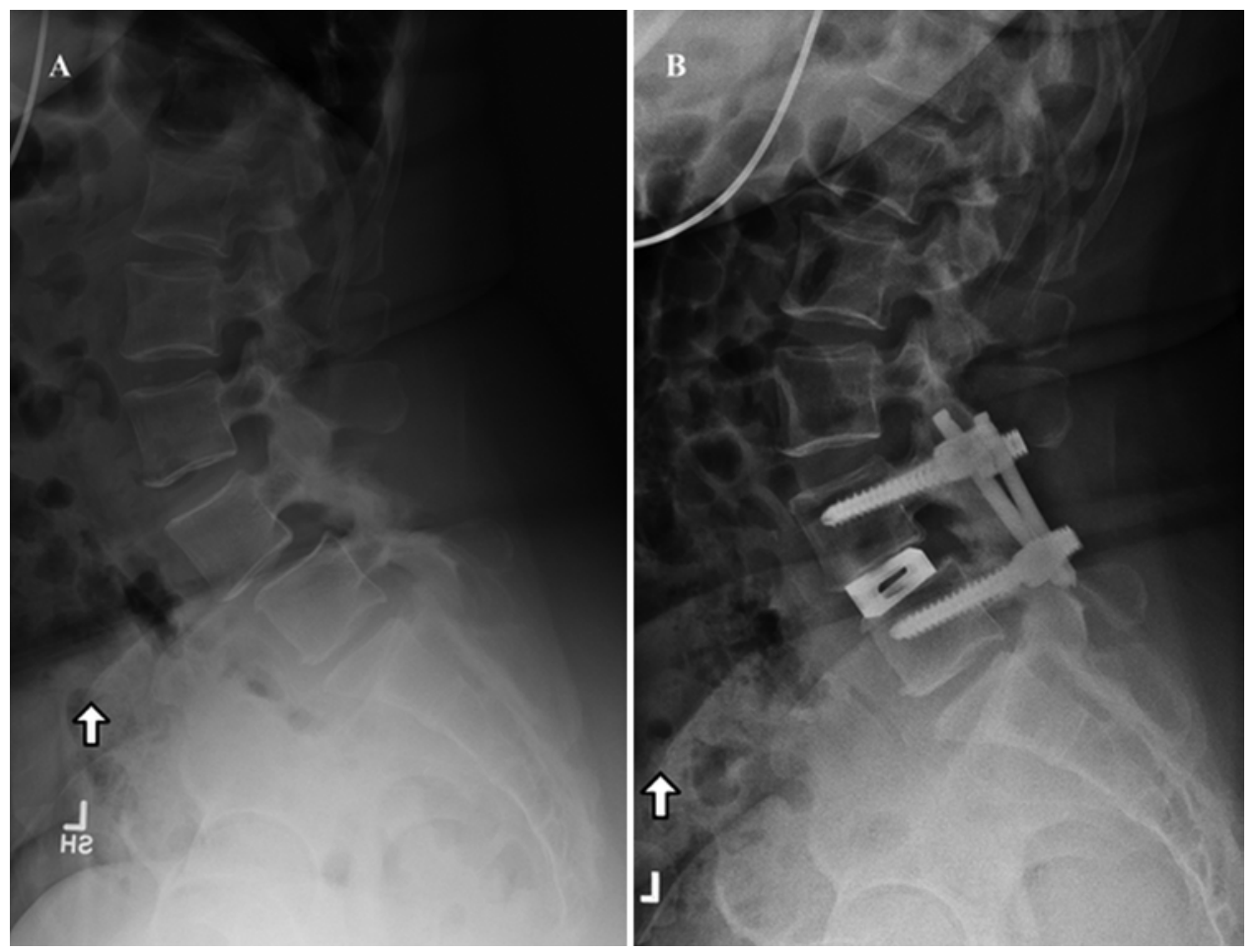

FIG. 1. Preoperative (A) and postoperative (B) lateral radiographs showing the reduction of a Grade 2 spondylolisthesis after LLIF and placement of supplemental posterior instrumentation. 


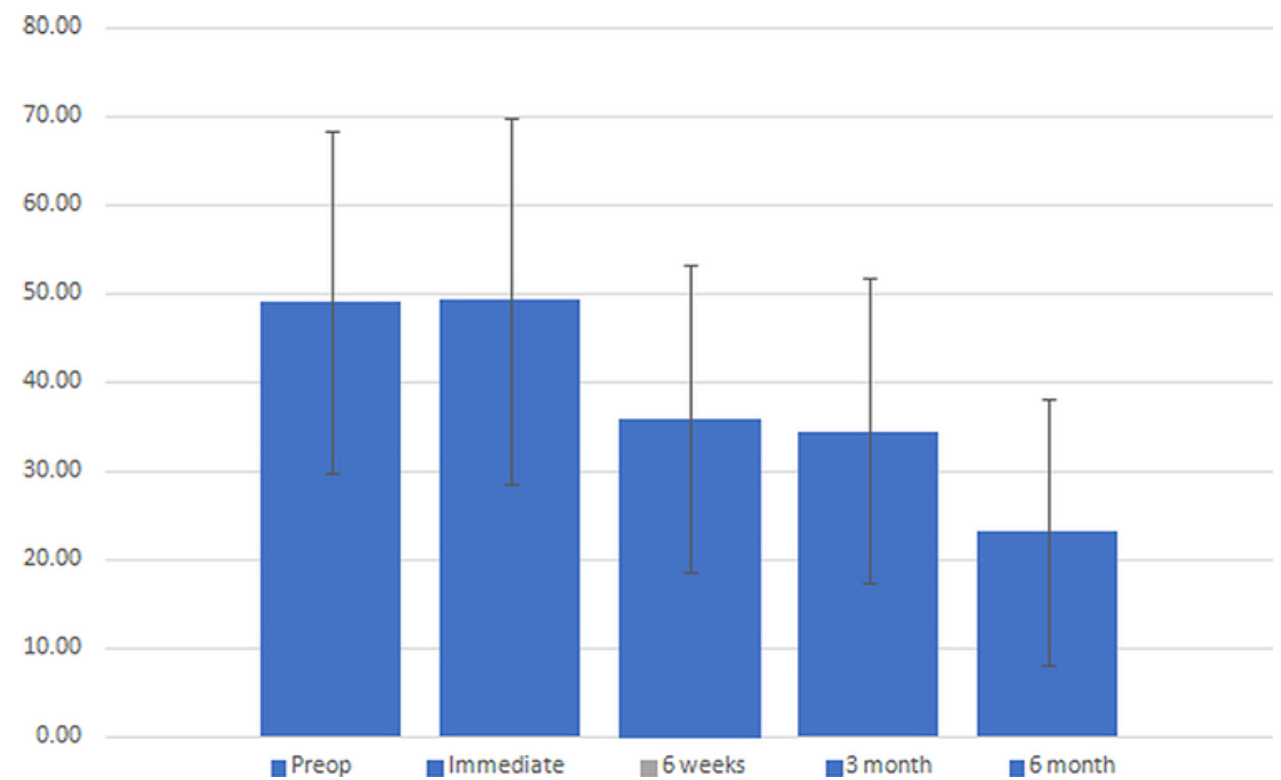

FIG. 2. Bar graph showing the mean ODI scores with standard deviations preoperatively, and at 2-week (Immediate), 6-week, 3-month, and 6-month postoperative intervals.

The mean SF-12 Mental Component Summary (MCS) and Physical Component Summary (PCS) scores improved after 6 weeks. At the time of the 3-month follow-up, PCS scores improved by 3.1 points and MCS scores improved by 4.8 points. At the 6-month follow-up, the mean PCS and MCS scores improved by 5.4 points and 4.7 points, respectively (Figs. 3 and 4). As with the ODI outcome, 12-month data were excluded because of a paucity of data, as they were only available for 2 patients.

\section{Discussion}

Lateral lumbar interbody fusion is a minimally invasive alternative to conventional fusion techniques that allows access to the intervertebral disc space without disrupting the anterior longitudinal ligament or posterior facets. The main aim of LLIF surgery is to restore disc space height with subsequent reduction of pain and improvement in disability. ${ }^{13}$ In traditional LLIF procedures, the psoas major muscle is split while the patient is in the direct lateral position, and an interbody graft is inserted between the vertebral apophyseal rings at one or more lumbar levels. This procedure has been successfully used for obtaining effective reduction and favorable alignment through a minimally disruptive approach and indirect decompression of the spinal canal and intervertebral foramen. ${ }^{2,21,25}$ A minimally invasive LLIF approach has been reported to reduce postoperative pain, tissue trauma, and operative and recovery times, thereby resulting in shorter hospital stays and brisker overall symptom improvement compared with traditional approaches. ${ }^{22,25}$

Neurological complications associated with lateral approaches are frequently discussed in the literature .,18 Previous anatomical and radiographic evaluations have identified that the lumbar plexus migrates dorsally to ventrally from L2 -3 as the lumbosacral plexus descends caudally to the L4-5 and L5-S1 levels., ${ }^{3,10,30}$ The underlying anatomi- cal arrangement can endanger the lumbosacral plexus in a transpsoas approach to the L4-5 level. ${ }^{27}$ Anterolisthesis of the L-4 vertebral body further dislocates the plexus even more ventrally, narrowing the safe zones of exposure to the L4-5 disc. ${ }^{30}$ In addition, sex-related anatomical variability can also make surgical exposure more difficult at L4-5, particularly in men with a large psoas muscle and a high iliac crest. ${ }^{29}$

The rate of perioperative medical complications in this survey was low (5.6\%). However, the rate of anterior thigh numbness or dysesthesia was noted to be $33 \%$. A review of the literature suggested that this complication occurs with a frequency of between $0 \%$ and $75 \%$ of LLIF cases. ${ }^{6}$ It is the practice at our institution to perform a complete lowerextremity sensory evaluation to light touch and pinprick at each postoperative encounter in all patients who undergo lateral surgery. Five patients reported ipsilateral sensory loss to the anterior thigh. One patient experienced 4 weeks of quadriceps weakness, which resolved completely. All sensory and motor symptoms resolved, most by the 6-week postoperative time point. One patient experienced thigh dysesthesia contralateral to the exposure, which resolved 6 months postoperatively. Imaging performed in this patient did not reveal any compressive pathology due to a migrated disc fragment or osteophyte. Papanastassiou et al. reported 2 cases of contralateral femoral nerve compression symptoms after LLIF, but both were secondary to anatomical compression from either extruded disc or osteophytes displaced into the contralateral psoas. ${ }^{23}$ With regard to neuropathic symptoms after LLIF, we posit a theory that has often been reported in studies evaluating complication reporting. Complication incidence varies widely throughout the literature due to variance in both the actual definition of the event as well as the perioperative examiner's extent of clinical vigilance, standardized exploration, and focus on a specific event or set of events. ${ }^{4,20,34}$

Given the risk of neurological complications, several 


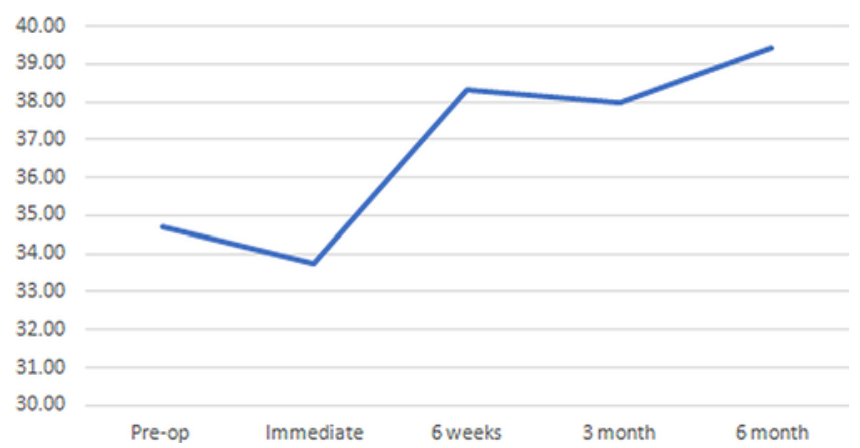

FIG. 3. Mean SF-12 PCS scores preoperatively, and at 2-week, 6-week, 3-month, and 6-month postoperative intervals.

strategies for reducing postoperative neural injury during LLIF have been presented in the literature. These range from novel finger electrode usage for intraoperative monitoring, ${ }^{19}$ triggered intermittent electromyography monitoring during psoas retraction, ${ }^{31}$ saphenous nerve somatosensory evoked potential monitoring 28 to complete avoidance of the transpsoas technique utilizing a prepsoas oblique lateral corridor for access to the disc space..$^{12}$ When employing LLIF for the indication of DS at L4-5, we feel that by strictly adhering to lateral access surgical principles, such as minimal table break, an initial look-and-see approach to the psoas, clear identification of the plexus, minimal expansion of the retractor, mobilization of any traversing sensory nerves, and psoas dilation times less than 20 minutes, ensure the lowest possible complication profile for both visceral and neural injuries even in the narrow safe zones experienced in accessing the L4-5 disc space in patients with DS.

In the current study, the mean ODI score improved more than 15 points at the 6-month time point. SF-12 MCS and PCS scores improved roughly $10 \%$ at the 3- and 6 -month time points. The FDA standards identify good to excellent operative outcomes when there is a 15-point improvement in ODI, plus maintenance or improvement in SF-36 score. ${ }^{9}$ To replicate the validity of the SF-36 with brevity and facilitate outcomes reporting at multiple time points, the SF-12 was developed and has been validated to report less than a $10 \%$ difference of the longer outcome measurement instrument across population studies. ${ }^{32}$ In the current study, we confirm the attainment of good to excellent clinical outcomes at the 3- and 6-month postoperative time points when treating Grade 1 and 2 DS with a transpsoas approach to the L4-5 disc space utilizing the FDA metrics.

Limitations of the current study include its relatively small sample size and the lack of 2- to 5-year follow-up data. These longer-term data are necessary for determining pseudarthrosis rates and for any meaningful comparison regarding adjacent-segment disease. As previously reported, given that this is a retrospective evaluation, complications are generally underreported compared with those of a true prospective study with a wide definition of a complication. ${ }^{4,20}$ In addition, the demographics in this patient survey were skewed to older and more obese patients, with a mean age of 64 years and average body mass index of $34.1 \mathrm{~kg} / \mathrm{m}^{2}$. Given this higher risk in our population, the

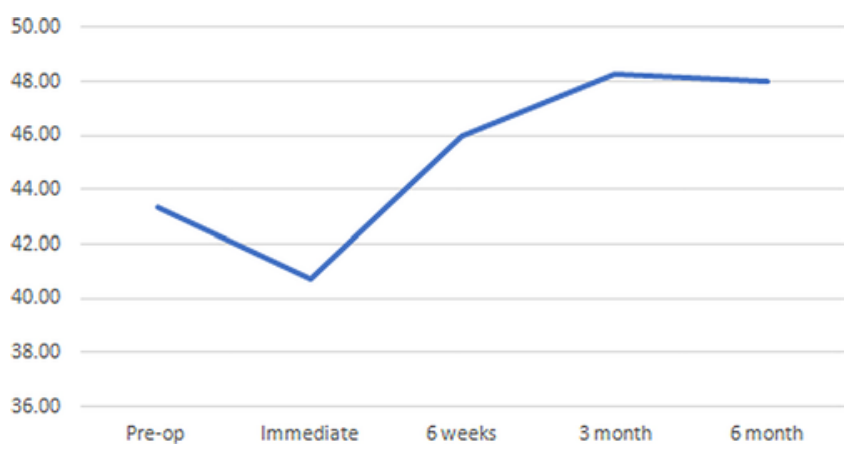

FIG. 4. Mean SF-12 MCS scores preoperatively, and at 2-week, 6-week, 3-month, and 6-month postoperative intervals.

data and outcomes may not be entirely comparable to a study with younger, leaner patients.

\section{Conclusions}

LLIF is a safe and effective treatment for patients with Grades 1 and 2 spondylolisthesis at L4-5. The use of this surgical approach provides a minimally invasive solution that offers favorable clinical and radiological outcomes with low rates of postoperative complications. However, the difficulty of exposure and the narrow neural safe zones mandate careful adherence to the lateral surgical technique and a detailed understanding of regional anatomy.

\section{References}

1. Ahmadian A, Verma S, Mundis GM Jr, Oskouian RJ Jr, Smith DA, Uribe JS: Minimally invasive lateral retroperitoneal transpsoas interbody fusion for L4 5 spondylolisthesis: clinical outcomes. J Neurosurg Spine 19:314-320, 2013

2. Alimi M, Hofstetter CP, Cong GT, Tsiouris AJ, James AR, Paulo D, et al: Radiological and clinical outcomes following extreme lateral interbody fusion. J Neurosurg Spine 20:623-635, 2014

3. Benglis DM, Vanni S, Levi AD: An anatomical study of the lumbosacral plexus as related to the minimally invasive transpsoas approach to the lumbar spine. J Neurosurg Spine 10:139-144, 2009

4. Campbell PG, Malone J, Yadla S, Chitale R, Nasser R, Maltenfort MG, et al: Comparison of ICD-9-based, retrospective, and prospective assessments of perioperative complications: assessment of accuracy in reporting. J Neurosurg Spine 14:16-22, 2011

5. Epstein NE: Extreme lateral lumbar interbody fusion: do the cons outweigh the pros? Surg Neurol Int 7 (Suppl 25):S692-S700, 2016

6. Epstein NE: High neurological complication rates for extreme lateral lumbar interbody fusion and related techniques: a review of safety concerns. Surg Neurol Int 7 (Suppl 25):S652-S655, 2016

7. Fogel GR, Turner AW, Dooley ZA, Cornwall GB: Biomechanical stability of lateral interbody implants and supplemental fixation in a cadaveric degenerative spondylolisthesis model. Spine (Phila Pa 1976) 39:E1138-E1146, 2014

8. Ghogawala Z, Dziura J, Butler WE, Dai F, Terrin N, Magge $\mathrm{SN}$, et al: Laminectomy plus fusion versus laminectomy alone for lumbar spondylolisthesis. N Engl J Med 374:14241434,2016

9. Glassman S, Gornet MF, Branch C, Polly D Jr, Peloza J, Schwender JD, et al: MOS Short Form 36 and Oswestry Dis- 
ability Index outcomes in lumbar fusion: a multicenter experience. Spine J 6:21-26, 2006

10. Hu WK, He SS, Zhang SC, Liu YB, Li M, Hou TS, et al: An MRI study of psoas major and abdominal large vessels with respect to the X/DLIF approach. Eur Spine J 20:557-562, 2011

11. Isaacs RE, Sembrano JN, Tohmeh AG: Two-year comparative outcomes of MIS lateral and MIS transforaminal interbody fusion in the treatment of degenerative spondylolisthesis: part II: radiographic findings. Spine (Phila Pa 1976) 41 (Suppl 8):S133-S144, 2016

12. Jin J, Ryu KS, Hur JW, Seong JH, Kim JS, Cho HJ: Comparative study of the difference of perioperative complication and radiologic results: MIS-DLIF (minimally invasive direct lateral lumbar interbody fusion) versus MIS-OLIF (minimally invasive oblique lateral lumbar interbody fusion). Clin Spine Surg [epub ahead of print], 2017

13. Johnson RD, Valore A, Villaminar A, Comisso M, Balsano M: Pelvic parameters of sagittal balance in extreme lateral interbody fusion for degenerative lumbar disc disease. J Clin Neurosci 20:576-581, 2013

14. Kepler CK, Bogner EA, Herzog RJ, Huang RC: Anatomy of the psoas muscle and lumbar plexus with respect to the surgical approach for lateral transpsoas interbody fusion. Eur Spine J 20:550-556, 2011

15. Khajavi K, Shen A, Hutchison A: Substantial clinical benefit of minimally invasive lateral interbody fusion for degenerative spondylolisthesis. Eur Spine J 24 (Suppl 3):314-321, 2015

16. Lee CW, Yoon KJ, Ha SS: Which approach is advantageous to preventing the development of adjacent segment disease? Comparative analysis of 3 different lumbar interbody fusion techniques (ALIF, LLIF, and PLIF) in L4-5 spondylolisthesis. World Neurosurg 105:612-622, 2017

17. Martin CR, Gruszczynski AT, Braunsfurth HA, Fallatah SM, O'Neil J, Wai EK: The surgical management of degenerative lumbar spondylolisthesis: a systematic review. Spine (Phila Pa 1976) 32:1791-1798, 2007

18. Moller DJ, Slimack NP, Acosta FL Jr, Koski TR, Fessler RG, Liu JC: Minimally invasive lateral lumbar interbody fusion and transpsoas approach-related morbidity. Neurosurg Focus 31(4): $\mathrm{E} 4,2011$

19. Narita W, Takatori R, Arai Y, Nagae M, Tonomura H, Hayashida T, et al: Prevention of neurological complications using a neural monitoring system with a finger electrode in the extreme lateral interbody fusion approach. J Neurosurg Spine 25:456-463, 2016

20. Nasser R, Yadla S, Maltenfort MG, Harrop JS, Anderson DG, Vaccaro AR, et al: Complications in spine surgery. J Neurosurg Spine 13:144-157, 2010

21. Oliveira L, Marchi L, Coutinho E, Pimenta L: A radiographic assessment of the ability of the extreme lateral interbody fusion procedure to indirectly decompress the neural elements. Spine (Phila Pa 1976) 35 (26 Suppl):S331-S337, 2010

22. Ozgur BM, Aryan HE, Pimenta L, Taylor WR: Extreme lateral interbody fusion (XLIF): a novel surgical technique for anterior lumbar interbody fusion. Spine J 6:435-443, 2006

23. Papanastassiou ID, Eleraky M, Vrionis FD: Contralateral femoral nerve compression: an unrecognized complication after extreme lateral interbody fusion (XLIF). J Clin Neurosci 18:149-151, 2011

24. Park DK, Lee MJ, Lin EL, Singh K, An HS, Phillips FM: The relationship of intrapsoas nerves during a transpsoas approach to the lumbar spine: anatomic study. J Spinal Disord Tech 23:223-228, 2010
25. Pereira EA, Farwana M, Lam KS: Extreme lateral interbody fusion relieves symptoms of spinal stenosis and low-grade spondylolisthesis by indirect decompression in complex patients. J Clin Neurosci 35:56-61, 2017

26. Resnick DK, Watters WC III, Sharan A, Mummaneni PV, Dailey AT, Wang JC, et al: Guideline update for the performance of fusion procedures for degenerative disease of the lumbar spine. Part 9: lumbar fusion for stenosis with spondylolisthesis. J Neurosurg Spine 21:54-61, 2014

27. Rodgers WB, Lehmen JA, Gerber EJ, Rodgers JA: Grade 2 spondylolisthesis at L4-5 treated by XLIF: safety and midterm results in the "worst case scenario". Sci World J 2012:356712, 2012

28. Silverstein J, Mermelstein L, DeWal H, Basra S: Saphenous nerve somatosensory evoked potentials: a novel technique to monitor the femoral nerve during transpsoas lumbar lateral interbody fusion. Spine (Phila Pa 1976) 39:1254-1260, 2014

29. Sofianos DA, Briseño MR, Abrams J, Patel AA: Complications of the lateral transpsoas approach for lumbar interbody arthrodesis: a case series and literature review. Clin Orthop Relat Res 470:1621-1632, 2012

30. Uribe JS, Arredondo N, Dakwar E, Vale FL: Defining the safe working zones using the minimally invasive lateral retroperitoneal transpsoas approach: an anatomical study. $\mathbf{J}$ Neurosurg Spine 13:260-266, 2010

31. Uribe JS, Isaacs RE, Youssef JA, Khajavi K, Balzer JR, Kanter AS, et al: Can triggered electromyography monitoring throughout retraction predict postoperative symptomatic neuropraxia after XLIF? Results from a prospective multicenter trial. Eur Spine J 24 (Suppl 3):378-385, 2015

32. Ware J Jr, Kosinski M, Keller SD: A 12-Item Short-Form Health Survey: construction of scales and preliminary tests of reliability and validity. Med Care 34:220-233, 1996

33. Weinstein JN, Lurie JD, Tosteson TD, Hanscom B, Tosteson AN, Blood EA, et al: Surgical versus nonsurgical treatment for lumbar degenerative spondylolisthesis. N Engl J Med 356:2257-2270, 2007

34. Yadla S, Malone J, Campbell PG, Maltenfort MG, Harrop JS, Sharan AD, et al: Early complications in spine surgery and relation to preoperative diagnosis: a single-center prospective study. J Neurosurg Spine 13:360-366, 2010

\section{Disclosures}

Drs. Nunley, Kerr, Utter, Stone, and Ms. Frank: support of nonstudy-related clinical or research support from K2M and Spineology. Dr. Campbell: consultant for 4 Web. Dr. Nunley: consultant for K2M; direct stock ownership in Amedica, Paradigm, and Spineology; patent holder with K2M, and LDR; and speakers bureau for K2M and LDR.

\section{Author Contributions}

Conception and design: Campbell. Acquisition of data: Campbell, Nunley, Frank. Analysis and interpretation of data: Campbell. Drafting the article: Campbell. Critically revising the article: Frank, Stone. Reviewed submitted version of manuscript: all authors. Approved the final version of the manuscript on behalf of all authors: Campbell.

\section{Correspondence}

Peter G. Campbell: Spine Institute of Louisiana, Shreveport, LA. pcampbell@louisianaspine.org. 